\title{
SEARCH FOR NEUTRINO MASS AND DARK MATTER IN UNDERGROUND EXPERIMENTS
}

\author{
H.V. KLAPDOR-KLEINGROTHAUS \\ Max-Planck-Institut für Kernphysik, \\ P.O. Box 1039 80, D-69029 Heidelberg, Germany \\ Spokesman of HEIDELBERG-MOSCOW and GENIUS Collaborations \\ E-mail: klapdor@gustav.mpi-hd.mpg, \\ Home-page: http://www.mpi-hd.mpg.de.non_acc
}

\begin{abstract}
Search for the neutrino mass and for cold dark matter in the Universe are at present two of the most exiting fields of particle physics and cosmology. This lecture will restrict itself on the search for neutralinos as cold dark matter, and for the absolute scale of the masses of neutrinos, which are the favoured hot dark matter candidates.
\end{abstract}

\section{Introduction}

In this lecture we shall discuss two central problems of particle physics and cosmology. The neutrino mass is one of the key quantities in grand unified theories, and at the same time candidate for hot dark matter in the Universe. Supersymmetry is regarded as the most natural extension of present particle physics theories. The lighest SUSY particle, usually assumed to be the neutralino, is the favored candidate for cold dark matter. We shall discuss here the search for the neutrino mass, concentrating on double beta decay, and the search for neutralinos, in underground experiments.

The neutrino oscillation interpretation of the atmospheric and solar neutrino data, deliver a strong indication for a non-vanishing neutrino mass. While such kind of experiments yields information on the difference of squared neutrino mass eigenvalues and on mixing angles, they cannot fix the absolute scale of the neutrino mass. Information from double beta decay experiments

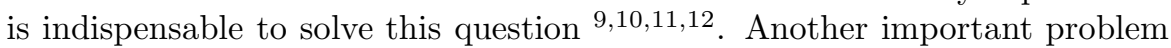
is that of the fundameptal character of the neutrino, whether it is a Dirac or a Majorana particle 23. Neutrinoless double beta decay could answer also this question. Perhaps the main question, which can be investigated by double beta decay with high sensitivity, is that of lepton number conservation or non-conservation.

The physics motivations to search for dark matter are manyfold.

Recent investigation of the cosmic microwave background radiation (MAXIMA, BOOMERANG, DASI) together with large scale structure results fix $\Omega_{\Lambda}+\Omega_{m} \sim 1$, where $\Omega_{\Lambda} \quad\left(=\rho_{\Lambda} / \rho_{c}\right)$ stands the for dark energy and

HVKK-Triest01-Prepr: submitted to World Scientific on November 10, 20181 
$\Omega_{m}$ for matter. With the early nucleosynthesis constraint of $\Omega_{\text {bar }} \sim 0.04$ the need for non-baryonic dark matter is evident. This is true even for our galaxy, since MACHOs represent only a small fraction of galactic dark matter 62 .

Although there exist other candidates such as axions 63 , gravitinos, etc., neutralinos seem to be the favored candidates at present.

Hot dark matter, according to CMB and LSS (Redshift-Survey) results still contributes up to $38 \%$ of the dark matter 8 . This corresponds to a sum of neutrino masses $<5.5 \mathrm{eV}$.

Our present picture of the mass/energy distribution in the Universe is as given in Fig.17.

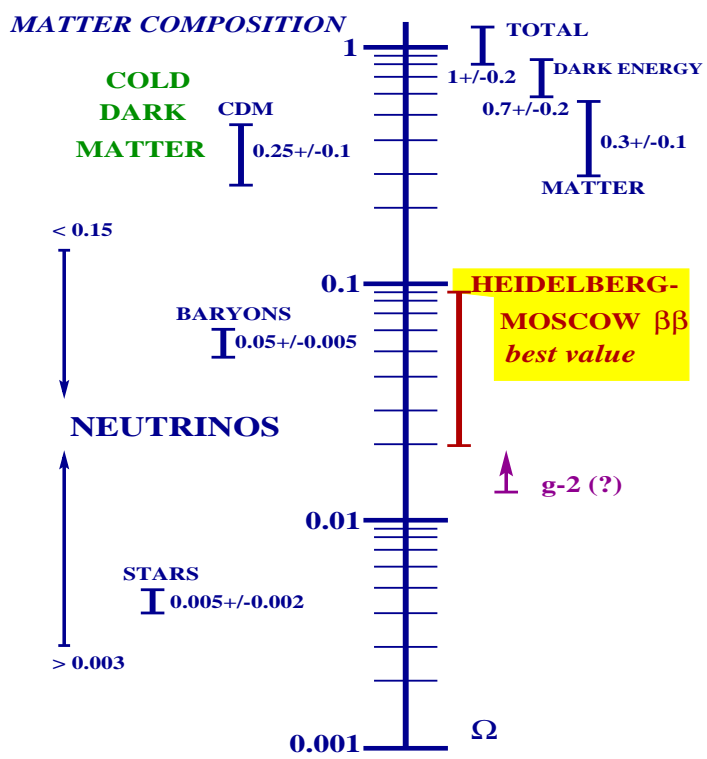

Figure 1. Our present understanding of the mass composition of the Universe.

In section 2 , we discuss double beta decay, including in section 2.2 the expectation from neutrino oscillation experiments, and in section 2.3 the recent evidence for the neutrinoless decay mode from the HEIDELBERG-MOSCOW experiment. In section 2.4 we discuss the future of the field.

In section 3 we shall discuss dark matter search. We start in 3.1 with the expectation from SUSY models and then discuss in section 3.2 the present status of cold dark matter experiments - including the important indication from the DAMA experiment - and its future. In section 3.3 we give some comments to neutrinos as hot dark matter. In section 4 we give a conclusion.

HVKK-Triest01-Prepr: submitted to World Scientific on November 10, 20182 


\section{Neutrino Masses and Double Beta Decay}

\subsection{General}

Double beta decay, the rarest known nuclear decay process, can occur in different modes:

$$
\begin{array}{ll}
2 \nu \beta \beta-\text { decay : } & A(Z, N) \rightarrow A(Z+2, N-2)+2 e^{-}+2 \bar{\nu}_{e} \\
0 \nu \beta \beta-\text { decay : } & A(Z, N) \rightarrow A(Z+2, N-2)+2 e^{-} \\
0 \nu(2) \chi \beta \beta \text { - decay }: & A(Z, N) \rightarrow A(Z+2, N-2)+2 e^{-}+(2) \chi
\end{array}
$$

While the two-neutrino mode (1) is allowed by the Standard Model of particle physics, the neutrinoless mode $(0 \nu \beta \beta)$ (2) requires violation of lepton number $(\Delta \mathrm{L}=2)$. This mode is possible only, if the neutrino is a Majorana particle, i.e. the neutrino is its ownantiparticle (E. Majorapa $f_{\text {B Bacah }}$ 3, for subsequent works we refer to 0 19, for some reviews see 14, 15, 16, $17,12,18)$. First calculations of $\mathrm{g} \beta \beta$ decay based on the Majorana theory have been done by W.H. Furry $\mathrm{t}$.

Neutrinoless double beta decay can not only probe a Majorana neutrino mass, but various new physics scenarios beyond the Standard Model, such as $\mathrm{R}$-parity violating supersymmetric models, R-parity conserving SUSY models, leptoquarks, violation of Lorentz-invariance, and compositeness (for a review see 12, $(2,41)$. Any theory containing lepton number violating interactions can in principle lead to this process allowing to obtain information on the specific underlying theory. The experimental signature of the neutrinoless mode is a peak at the $\mathrm{Q}$-value of the decay.

The unique feature of neutrinoless double beta decay is that a measured half-life allows to deduce information on the effective Majorana peutrino mass $\langle m\rangle$, which is a superposition of neutrino mass eigenstates: 14,15

$$
\begin{aligned}
{\left[T_{1 / 2}^{0 \nu}\left(0_{i}^{+} \rightarrow 0_{f}^{+}\right)\right]^{-1}=} & C_{m m} \frac{\langle m\rangle^{2}}{m_{e}^{2}}+C_{\eta \eta}\langle\eta\rangle^{2}+C_{\lambda \lambda}\langle\lambda\rangle^{2}+C_{m \eta}\langle\eta\rangle \frac{\langle m\rangle}{m_{e}} \\
& +C_{m \lambda}\langle\lambda\rangle \frac{\left\langle m_{\nu}\right\rangle}{m_{e}}+C_{\eta \lambda}\langle\eta\rangle\langle\lambda\rangle, \\
\langle m\rangle & =\left|m_{e e}^{(1)}\right|+e^{i \phi_{2}}\left|m_{e e}^{(2)}\right|+e^{i \phi_{3}}\left|m_{e e}^{(3)}\right|,
\end{aligned}
$$

where $m_{e e}^{(i)} \equiv\left|m_{e e}^{(i)}\right| \exp \left(i \phi_{i}\right)(i=1,2,3)$ are the contributions to $\langle m\rangle$ from individual mass eigenstates, with $\phi_{i}$ denoting relative Majorana phases connected with $\mathrm{CP}$ violation, and $C_{m m}, C_{\eta \eta}, \ldots$ denote nuclear matrix elements,

HVKK-Triest01-Prepr: submitted to World Scientific on November 10, 20183 
which can be calculated, (see, e.g. 23, for a review see e.g. 24, 22, 15, 19, 44 ). Ignoring contributions from right-handed weak currents on the right-hand side of eq.(1), only the first term remains.

\subsection{Allowed Ranges of $\langle m\rangle$ by $\nu$ Oscillation Experiments}

The observable of double beta decay

$$
\langle m\rangle=\left|m_{e e}^{(1)}\right|+e^{i \phi_{2}}\left|m_{e e}^{(2)}\right|+e^{i \phi_{3}}\left|m_{e e}^{(3)}\right|,
$$

with $U_{e i}$ denoting elements of the neutrino mixing matrix, $m_{i}$ neutrino mass eigenstates, and $\phi_{i}$ relative Majorana $\mathrm{CP}$ phases, can be written in terms of oscillation parameters 10.11

$$
\begin{aligned}
& \left|m_{e e}^{(1)}\right|=\left|U_{e 1}\right|^{2} m_{1}, \\
& \left|m_{e e}^{(2)}\right|=\left|U_{e 2}\right|^{2} \sqrt{\Delta m_{21}^{2}+m_{1}^{2}}, \\
& \left|m_{e e}^{(3)}\right|=\left|U_{e 3}\right|^{2} \sqrt{\Delta m_{32}^{2}+\Delta m_{21}^{2}+m_{1}^{2}} .
\end{aligned}
$$

The effective mass $\langle m\rangle$ is related with the half-life for $0 \nu \beta \beta$ decay via $\left(T_{1 / 2}^{0 \nu}\right)^{-1} \sim\left\langle m_{\nu}\right\rangle^{2}$, and for the limit on $T_{1 / 2}^{0 \nu}$ deducible in an experiment we have $T_{1 / 2}^{0 \nu} \sim a \sqrt{\frac{M t}{\Delta E B}}$. Here $a$ is the isotopical abundance of the $\beta \beta$ emitter; $M$ is the active detector mass; $t$ is the measuring time; $\Delta E$ is the energy resolution; $B$ is the background count rate.

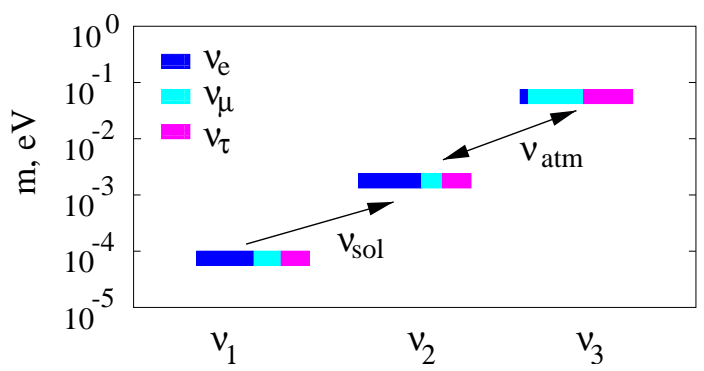

Figure 2. Neutrino masses and mixings in the scheme with mass hierarchy. Coloured bars correspond to flavor admixtures in the mass eigenstates $\nu_{1}, \nu_{2}, \nu_{3}$. The quantity $\langle m\rangle$ is determined by the dark blue bars denoting the admixture of the electron neutrino $U_{e i}$.

Neutrino oscillation experiments fix or restrict some of the parameters in (1)-(3), e.g. in the case of normal hierarchy solar neutrino experiments yield $\Delta m_{21}^{2},\left|U_{e 1}\right|^{2}=\cos ^{2} \theta_{\odot}$ and $\left|U_{e 2}\right|^{2}=\sin ^{2} \theta_{\odot}$. Atmospheric neutrinos fix $\Delta m_{32}^{2}$, and experiments like CHOOZ, looking for $\nu_{e}$ disappearance restrict

HVKK-Triest01-Prepr: submitted to World Scientific on November 10, 20184 
$\left|U_{e 3}\right|^{2}$. The phases $\phi_{i}$ and the mass of the lightest neutrino, $m_{1}$ are free parameters. Double beta decay can fix the parameter $\mathrm{m}_{1}$ and thus the absolute mass scale. The expectations for $\langle m\rangle$ from oscillation experiments in different neutrino mass scenarios have been carefully analyzed in 20.11 . In sections 2.2.1. to 2.2.3. we give some examples.

\subsubsection{Hierarchical Spectrum $\left(m_{1} \ll m_{2} \ll m_{3}\right)$}

In hierarchical spectra (Fig. 2), motivated by analogies with the quark sector and the simplest see-saw models, the main contribution comes from $m_{2}$ or $m_{3}$. For the large mixing angle (LMA) MSW solution which is favored at present for the solar neutrino problem (see 32), the contribution of $m_{2}$ becomes dominant in the expression for $\langle m\rangle$, and

$$
\langle m\rangle \simeq m_{e e}^{(2)}=\frac{\tan ^{2} \theta}{1+\tan ^{2} \theta} \sqrt{\Delta m_{\odot}^{2}} .
$$

In the region allowed at $90 \%$ C.L. by Superkamiokande according to 33 , the prediction for $\langle m\rangle$ becomes

$$
\langle m\rangle=(1 \div 3) \cdot 10^{-3} \mathrm{eV} .
$$

The prediction extends to $\langle m\rangle=10^{-2} \mathrm{eV}$ in the $99 \%$ C.L. range (Fig. 3).

\subsubsection{Inverse Hierarchy $\left(m_{3} \approx m_{2} \gg m_{1}\right)$}

In inverse hierarchy scenarios (Fig. (4) the heaviest state with mass $m_{3}$ is mainly the electron neutrino, its mass being determined by atmospheric neutrinos, $m_{3} \simeq \sqrt{\Delta m_{\mathrm{atm}}^{2}}$. For the LMA MSW solution one finds 11

$$
\langle m\rangle=(1 \div 7) \cdot 10^{-2} \mathrm{eV} \text {. }
$$

\subsubsection{Degenerate Spectrum $\left(m_{1} \simeq m_{2} \simeq m_{3} \sim 0.1 \mathrm{eV}\right)$}

In degenerate scenarios (fig. (1) the contribution of $m_{3}$ is strongly restricted by CHOOZ. The main contributions come from $m_{1}$ and $m_{2}$, depending on their admixture to the electron flavors, which is determined by the solar neutrino solution. We find 11

$$
m_{\min }<\langle m\rangle<m_{1} \quad \text { with } \quad\left\langle m_{\min }\right\rangle=\left(\cos ^{2} \theta_{\odot}-\sin ^{2} \theta_{\odot}\right) m_{1} .
$$

This leads for the LMA solution to $\langle m\rangle=(0.25 \div 1) \cdot m_{1}$, the allowed range corresponding to possible values of the unknown Majorana $\mathrm{CP}$-phases.

After these examples we give a summary of our analysis 10,11 of the $\langle m\rangle$ allowed by $\nu$ oscillation experiments for neutrino mass models in the presently

HVKK-Triest01-Prepr: submitted to World Scientific on November 10, 20185 


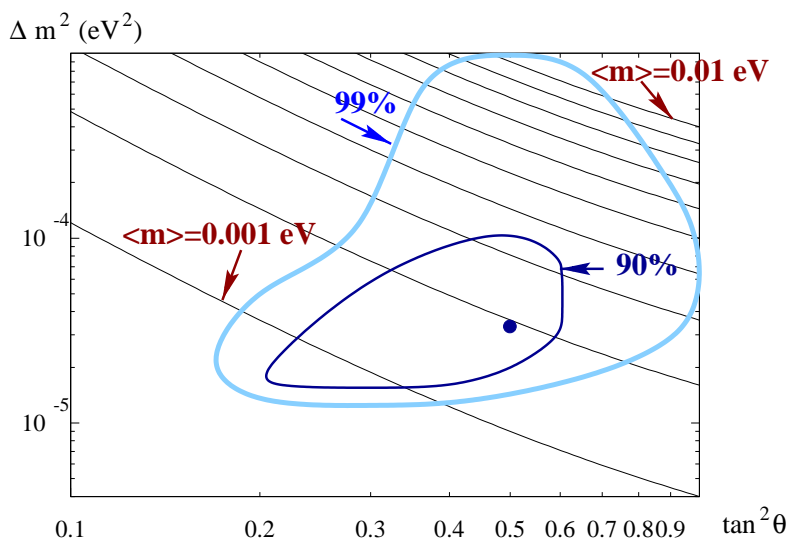

Figure 3. Double beta decay observable $\langle m\rangle$ and oscillation parameters in the case of the MSW large mixing angle solution of the solar neutrino deficit, where the dominant contribution to $\langle m\rangle$ comes from the second state. Shown are lines of constant $\langle m\rangle$, the lowest line corresponding to $\left\langle m_{\nu}\right\rangle=0.001 \mathrm{eV}$, the upper line to $0.01 \mathrm{eV}$. The inner and outer closed line show the regions allowed by present solar neutrino experiments with $90 \%$ C.L. and $99 \%$ C.L., respectively. Double beta decay with sufficient sensitivity could check the LMA MSW solution. Complementary information could be obtained from the search for a day-night effect and spectral distortions in future solar neutrino experiments as well as a disappearance signal in KAMLAND [from 11].
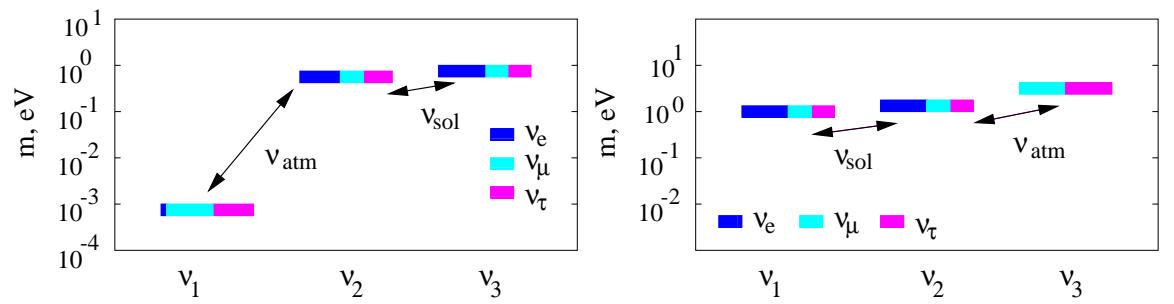

Figure 4. Left: Neutrino masses and mixing in the inverse hierarchy scenario. Right: Neutrino masses and mixings in the degenerate scheme.

favored scenarios, in Fig. 16. The size of the bars corresponds to the uncertainty in mixing angles and the unknown Majorana CP-phases.

HVKK-Triest01-Prepr: submitted to World Scientific on November 10, 20186 


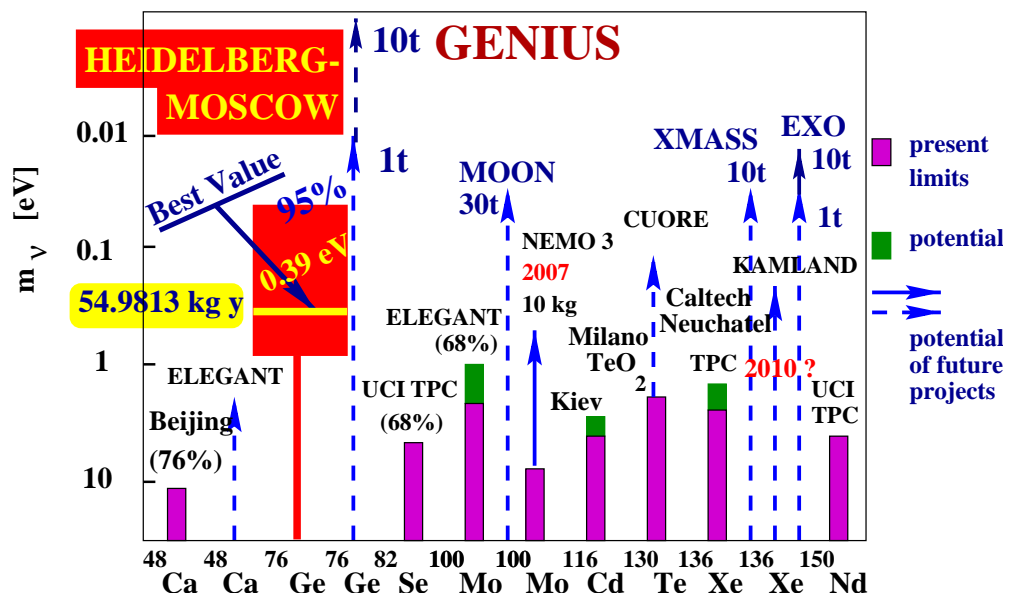

Figure 5. Present sensitivity, and expectation for the future, of the most promising $\beta \beta$ experiments. Given are limits for $\langle p\rangle\rangle$, except for the HEIDELBERG-MOSCOW experiment where the recently observed value 1 is given (95\% c.l. range), and the best value. Framed parts of the bars: present status; not framed parts: expectation for running experiments; solid and das limes: experiments under construction or proposed, respectively. For references see $12,85,03$.

\subsection{Status of $\beta \beta$ Research and Evidence for the Neutrinoless Decay Mode}

The status of present double beta experiments is shown in Fig. 5 and is extensively discussed in 12 . The HEIDELBERG-MOSCOW experiment using the largest source strength of $11 \mathrm{~kg}$ of enriched ${ }^{76} \mathrm{Ge}$ in form of five HP Gedetectors is running since August 1990 in the Gran-Sasso underground laboratory 34. 39. 12.1. B, and is since long time the most sensitive one. We communicate here the status of the analysis of November 2001.

\subsubsection{Data from the HEIDELBERG-MOSCOW Experiment}

We have analysed 1 the data taken in the period August 1990 - May 2000 $(54.9813 \mathrm{kgy}$, or 723.44 mol-years and the data of single site events taken in the period Novemper 1995 - May 2000 with our methods of pulse shape analysis (PSA) 19.21.20, with various statistical methods, in particular with the Bayesian method (see, e.g. 22). This method is particularly suited for low counting rates, where the data follow a Poisson distribution, that cannot be approximated by a Gaussian.

This Bayesian procedure reproduces $\gamma$-lines at the position of known weak

HVKK-Triest01-Prepr: submitted to World Scientific on November 10, 20187 
lines from the decay of ${ }^{214} \mathrm{Bi}$ at $2010.7,2016.7,2021.8$ and $2052.9 \mathrm{keV} 25$. In addition, a linecentered at $2039 \mathrm{keV}$ shows up. This is compatible with the Q-value 27,26 of the double beta decay process. We emphasize, that at this energy no $\gamma$-line is expected according to Monte Carlo simulations of our experimental setup, and to the compilations in 2 . Therefore, on the Bayesian analysis yields a confidence level for a line to exist at $2039.0 \mathrm{keV}$ of $97 \%$ c.l. $(2.2 \sigma)$.

\subsection{2. $\beta \beta$ - Half-Life and Effective Neutrino Mass}

Under the assumption that the signal at $\mathrm{Q}_{\beta \beta}$ is not produced by a background line of at present unknown origin, we can translate the observed number of events into half-lifes for neutrinoless double beta decay.

In Table 1 we give the values obtained with the Bayesian method. Also given are the effective neutrino mass $\langle m\rangle$ deduced using matrix elements from 23 .

Table 1. Half-life for the neutrinoless decaynmode and deduced effective neutrino mass from the HEIDELBERG-MOSCOW experiment.

\begin{tabular}{c|c|c|c|c}
\hline \hline $\begin{array}{c}\text { Significan- } \\
\text { ce }[k g y]\end{array}$ & Detectors & $\mathrm{T}_{1 / 2}^{0 \nu} \mathrm{y}$ & $\langle m\rangle \mathrm{eV}$ & $\begin{array}{c}\text { Conf. } \\
\text { level }\end{array}$ \\
\hline 54.9813 & $1,2,3,4,5$ & $(0.80-35.07) \times 10^{25}$ & $(0.08-0.54)$ & $95 \%$ c.l. \\
54.9813 & $1,2,3,4,5$ & $(1.04-3.46) \times 10^{25}$ & $(0.26-0.47)$ & $68 \%$ c.l. \\
54.9813 & $1,2,3,4,5$ & $1.61 \times 10^{25}$ & 0.38 & Best Value \\
\hline 46.502 & $1,2,3,5$ & $(0.75-18.33) \times 10^{25}$ & $(0.11-0.56)$ & $95 \%$ c.l. \\
46.502 & $1,2,3,5$ & $(0.98-3.05) \times 10^{25}$ & $(0.28-0.49)$ & $68 \%$ c.l. \\
46.502 & $1,2,3,5$ & $1.50 \times 10^{25}$ & 0.39 & Best Value \\
\hline 28.053 & $2,3,5 \mathrm{SSE}$ & $(0.88-22.38) \times 10^{25}$ & $(0.10-0.51)$ & $90 \%$ c.l. \\
28.053 & $2,3,5 \mathrm{SSE}$ & $(1.07-3.69) \times 10^{25}$ & $(0.25-0.47)$ & $68 \%$ c.l. \\
28.053 & $2,3,5 \mathrm{SSE}$ & $1.61 \times 10^{25}$ & 0.38 & Best Value \\
\hline \hline
\end{tabular}

We derive from the data taken with $46.502 \mathrm{~kg}$ y the half-life $\mathrm{T}_{1 / 2}^{0 \nu}=(0.8-$ $18.3) \times 10^{25}$ y (95\% c.l.). The analysis of the other data sets, shown in Table 1, and in particular of the single site events data, which play an important role in our conclusion, confirm this result.

The result obtained is consistent with the limits given earlier by the HEIDELBERG-MOSCOW experiment 1 . It is also consistent with all other double beta experiments - which still reach less sensitivity. A second Ge-expe-

HVKK-Triest01-Prepr: submitted to World Scientific on November 10, $2018 \mathbf{8}$ 
riment which has stopped operation in 1999 after reaching a significance of $9 \mathrm{kgy} 57$ yields (if one believes their method of 'visual inspection' in their data analysis $)$ in a conservative analysis a limit of $\mathrm{T}_{1 / 2}^{0 \nu}>0.55 \times 10^{25} \mathrm{y}(90 \%$ c1.). (and not the value given in 57). The ${ }^{128} \mathrm{Te}$ geochemical experiment 51 yields $\left\langle m_{\nu}\right\rangle<1.1 \mathrm{eV}(68 \%$ c.l. $)$, the ${ }^{130} \mathrm{Te}$ cryogenic experiment yields $\left\langle m_{\nu}\right\rangle<1.8 \mathrm{eV} 53$, and the $\mathrm{Cd}_{5} \mathrm{WO}_{4}$ experiment $\left\langle m_{\nu}\right\rangle<2.6 \mathrm{eV}$, 52, all derived with the matrix elements of 23 to make the results comparable to the present value.

Concluding we obtain, with about $95 \%$ probability, first evidence for the neutrinoless double beta decay mode. As a consequence, with $95 \%$ confidence, lepton number is not conserved. Further the neutrino is a Majorana particle. The effective mass $\langle m\rangle$ is deduced to be $\langle m\rangle=(0.11-0.56) \mathrm{eV}$ (95\% c.l.), with best value of $0.39 \mathrm{eV}$. Allowing conservatively for an uncertainty of the nuclear matrix elements of $\pm 50 \%$ (for detailed discussions of thestafus of nuclear matrix elements we refer to 12 and references therein like 24.18 . 44 ) this range may widen to $\langle m\rangle=(0.05-0.84) \mathrm{eV}(95 \%$ c.l. $)$.

In this conclusion, it is assumed that contributions to $0 \nu \beta \beta$ decay from processes other than the exchange of a Majorana neutrino (see, e.g. 12,13 and references therein) are negligible.

\subsection{Future of $\boldsymbol{\beta} \boldsymbol{\beta}$ Experiments}

To improve the present sensitivity for the effective neutrino mass considerably, and to fix this quantity more accurately requires new experimental approaches, as discussed extensively in 12, $93,36,28,29,30$. Some of them are indicated in Figs. 5.16.

It has been pointed out earlier (see e.g. 12, 93, 36, 28, 30, 36), that of present generation experiments probably no one has a potential to probe $\langle m\rangle$ below (and perhaps even down to) the present HEIDELBERG-MOSCOW level (see Fig. 5).

The Milano cryogenic experiment using $\mathrm{TeO}_{2}$ bolometers improved their values for the $\langle m\rangle$ from $\beta \beta$ decay of ${ }^{130} \mathrm{Te}$, from $5.3 \mathrm{eV}$ in $1994 \mathrm{ed}$ to $1.8 \mathrm{eV}$ in 200053 . NEMO-III, originally aiming at a sensitivity of $0.1 \mathrm{eV}$, reduced their goals recently to $0.3 \div 0.7 \mathrm{eV}$ (see 55 ) (which is more consistent with estimates given by 54 ), to be reached in 6 years from starting of running, foreseen for the year 2002 .

\subsubsection{GENIUS and other Proposed Future Double Beta Experiments}

With the era of the HEIDELBERG-MOSCOW experiment the time of the small smart experiments is over.

HVKK-Triest01-Prepr: submitted to World Scientific on November 10, 20189 
To reach significantly larger sensitivity, $\beta \beta$ experiments have to become large. On the other hand source strengths of up to 10 tons of enriched material touch the world production limits. This means that the background has to be reduced by the order a factor of 1000 and more compared to that of the HEIDELBERG-MOSCOW experiment.

Table 2. Some key numbers of future double beta decay experiments (and of the

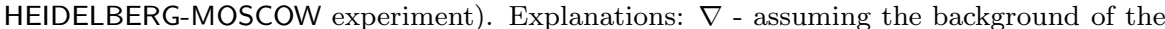
present pilot project. $* *$ - with matrix element from $23,24,45,46,47$ (see Table II in 35 ) and assuming an uncertainty of $\pm 50 \%$ of the nuclear matrix element. $\triangle$ this case shown to demonstrate the ultimate limit of such experiments. For details see 12

\begin{tabular}{|c|c|c|c|c|c|c|c|}
\hline $\begin{array}{c}\beta \beta- \\
\text { Isoto- } \\
\text { pe }\end{array}$ & Name & Status & $\begin{array}{c}\text { Mass } \\
\text { (ton- } \\
\text { nes })\end{array}$ & $\begin{array}{c}\text { Assumed } \\
\text { backgr. } \\
\dagger \text { events/ } \\
\text { kg y keV, } \\
\ddagger \text { events/kg } \\
\text { y FWHM, } \\
\text { * events } \\
\text { /yFWHM } \\
\end{array}$ & $\begin{array}{c}\text { Running } \\
\text { Time } \\
\text { (tonn. } \\
\text { years) }\end{array}$ & $\begin{array}{c}\text { Results } \\
\text { limit for } \\
0 \nu \beta \beta \\
\text { half-life } \\
\text { (years) }\end{array}$ & $\begin{array}{l}<m_{\nu}> \\
(\mathrm{eV})\end{array}$ \\
\hline${ }^{76} \mathrm{Ge}$ & 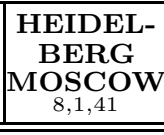 & $\begin{array}{l}\text { run- } \\
\text { ning } \\
\text { since } \\
1990 \\
\end{array}$ & $\begin{array}{l}0.011 \\
\text { (enri- } \\
\text { ched) }\end{array}$ & $\begin{array}{c}\dagger 0.06 \\
\\
\ddagger 0.24 \\
* 2 \\
\end{array}$ & $\begin{array}{c}54.98 \\
\mathrm{kgy}\end{array}$ & $\begin{array}{c}(0.8-18.3) \\
\text { x 10 } 10^{25} y \\
95 \% \text { c.l. } \\
\text { NOW !! } \\
\end{array}$ & $\begin{array}{c}(0.05-0.84) \\
\text { eV })^{* *} \\
95 \% \text { c.l. } \\
\text { NOW !! } \\
\end{array}$ \\
\hline${ }^{100} \mathrm{Mo}$ & NEMA III & $\begin{array}{c}\text { under } \\
\text { constr. } \\
\text { end2001? }\end{array}$ & $\begin{array}{l}\sim 0.01 \\
\text { (enri- } \\
\text {-ched) }\end{array}$ & $\begin{array}{c}\dagger \mathbf{0 . 0 0 0 5} \\
\ddagger 0.2 \\
* 2 \\
\end{array}$ & $\begin{array}{r}50 \\
\mathrm{~kg} \mathrm{y} \\
\end{array}$ & $10^{24}$ & $0.3-0.7$ \\
\hline${ }^{130} \mathrm{Te}$ & Cuppip $^{\nabla}$ & $\begin{array}{c}i d e a \\
\text { since1998 }\end{array}$ & $\begin{array}{c}0.75 \\
\text { (nat.) }\end{array}$ & $\begin{array}{c}\dagger 0.5 \\
\ddagger 4.5 / * 1000 \\
\end{array}$ & 5 & $9 \cdot 10^{24}$ & $0.2-0.5$ \\
\hline${ }^{130} \mathrm{Te}$ & qupt:56 & $\begin{array}{c}\text { idea } \\
\text { since1998 } \\
\end{array}$ & $\begin{array}{c}0.75 \\
\text { (nat.) }\end{array}$ & $\begin{array}{c}\dagger 0.005 \\
\ddagger 0.045 / * 45\end{array}$ & 5 & $9 \cdot 10^{25}$ & $0.07-0.2$ \\
\hline${ }^{100} \mathrm{Mo}$ & Nân & $\begin{array}{c}\text { idea } \\
\text { since1999 } \\
\end{array}$ & $\begin{array}{l}10 \text { (enr.) } \\
100 \text { (nat.) }\end{array}$ & $?$ & $\begin{array}{c}30 \\
300 \\
\end{array}$ & $?$ & 0.03 \\
\hline${ }^{116} \mathrm{Cd}$ & \begin{tabular}{|c|} 
CAMEOI \\
CAMEOII 60 \\
\end{tabular} & $\begin{array}{c}\text { idea } \\
\text { since2000 }\end{array}$ & \begin{tabular}{|c|}
0.65 \\
1 (enr.) \\
\end{tabular} & $\begin{array}{c}* 3 . \\
?\end{array}$ & $\begin{array}{l}5-8 \\
5-8 \\
\end{array}$ & $\begin{array}{l}10^{26} \\
10^{27} \\
\end{array}$ & $\begin{array}{l}0.06 \\
0.02 \\
\end{array}$ \\
\hline${ }^{136} \mathrm{Xe}$ & \begin{tabular}{|l|r|} 
EXO \\
49.50 \\
\end{tabular} & \begin{tabular}{|c|} 
Proposal \\
since \\
1999 \\
\end{tabular} & $\begin{array}{c}1 \\
10 \\
\end{array}$ & $\begin{array}{l}* 0.4 \\
* 0.6 \\
\end{array}$ & $\begin{array}{c}5 \\
10 \\
\end{array}$ & $\begin{array}{l}8.3 \cdot 10^{26} \\
1.3 \cdot 10^{28} \\
\end{array}$ & $\begin{array}{l}0.05-0.14 \\
0.01-0.04 \\
\end{array}$ \\
\hline${ }^{76} \mathrm{Ge}$ & $\begin{array}{c}\text { GENIUS } \\
\text { (2) }\end{array}$ & $\begin{array}{c}\text { under } \\
\text { constr. } \\
\text { end 2002 }\end{array}$ & $\begin{array}{l}11 \mathrm{~kg} \\
(\mathrm{enr} .)\end{array}$ & $\dagger 6 \cdot 10^{-3}$ & 3 & $1.6 \cdot 10^{26}$ & 0.15 \\
\hline${ }^{76} \mathrm{Ge}$ & GEN & $\begin{array}{l}\text { Pro- } \\
\text { posal } \\
\text { since } \\
1997 \\
\end{array}$ & $\begin{array}{c}1 \\
(\mathrm{enr} .) \\
1 \\
\end{array}$ & \begin{tabular}{|c|}
$\dagger 0.04 \cdot 10^{-3}$ \\
$\ddagger 0.15 \cdot 10^{-3}$ \\
$* 0.15$ \\
$* 1.5$ \\
\end{tabular} & 10 & $\begin{array}{l}5.8 \cdot 10^{27} \\
2 \cdot 10^{28} \\
\end{array}$ & \begin{tabular}{|l}
$0.02-0.05$ \\
$0.01-0.028$ \\
\end{tabular} \\
\hline${ }^{76} \mathrm{Ge}$ & 吉 & $\begin{array}{l}\text { Pro- } \\
\text { posal } \\
\text { since } \\
1997 \\
\end{array}$ & $\begin{array}{c}10 \\
\text { (enr.) }\end{array}$ & $\mid \begin{array}{c}\mathbf{0 . 1 5} \cdot 10^{-3} \\
0^{\triangle}\end{array}$ & $\begin{array}{l}10 \\
10\end{array}$ & $\begin{array}{c}6 \cdot 10^{28} \\
5.7 \cdot 10^{29}\end{array}$ & \begin{tabular}{|c|}
$0.006-$ \\
0.016 \\
$0.002-$ \\
0.0056 \\
\end{tabular} \\
\hline
\end{tabular}

HVKK-Triest01-Prepr: submitted to World Scientific on November 10, 201810 
Table 2 lists some key numbers for GENIUS2829. 36 , which was the first proposal for a third generation double beta experiment, and of some other proposals made after the GENIUS proposal. The potential of some of them is shown also in Fig. 16, and it is seen that not all of them will lead to large improvements in sensitivity. Among the latter is also the recently presented MAJORANA project 8 , which does not really apply a striking new strategy for background reduction, particularly also after it was found that the projected segmentation of detectors may not work (see Table 3 ).

Table 3. Some of the new projects under discussion for future double beta decay experiments (see ref.12).

\begin{tabular}{|c|c|c|c|c|}
\hline & NEV & PROJEC & $\overline{\mathrm{TS}}$ & \\
\hline & $\begin{array}{l}\text { BACKGROUND } \\
\text { REDUCTION }\end{array}$ & $\begin{array}{c}\text { MASS } \\
\text { INCREASE }\end{array}$ & $\begin{array}{c}\text { POTENTIAL } \\
\text { FOR DARK } \\
\text { MATTER }\end{array}$ & $\begin{array}{c}\text { POTENTIAL } \\
\text { FOR STLAAR } \\
\nu \mathrm{s}\end{array}$ \\
\hline GENIUS & + & + & + & $+^{*)}$ \\
\hline CUORE & $(+)$ & + & - & - \\
\hline MOON & $(+)$ & + & - & + \\
\hline EXO & + & + & - & - \\
\hline MAJORANA & - & + & - & - \\
\hline
\end{tabular}

For more recent information on XMASS, EXO, MOON experiments see the contributions of Y. Suzuki, G. Gratta and H. Ejiri in Ref. 59. The CAMEO project 60 in its now propagated variant GEM is nothing then a variant of GENWS (see below) put into the BOREXINO tank, at some later time. CUORE 21 has, with the complexity of cryogenic techniques, still to overcome serigus problems of background to enter into interesting regions of $\left\langle m_{\nu}\right\rangle$. EXO 49 needs still very extensive research and development to probe the applicability of the proposed detection method. In particular if it would be confirmed that tracks will be too short to be identified, it would act essentially only as a highly complicated calorimeter. In the GENIUS project a reduction by a factor of more than 1000 down to a background level of 0.1 events/tonne $\mathrm{y} \mathrm{keV}$ in the range of $0 \nu \beta \beta$ decay is planned to be reached by removing all material close to the detectors, and by using naked Germanium detectors in a large tank of liquid nitrogen. It has been shown that the detectors show excellent performance under such conditions 29. 38 . For technical questions and extensive Monte Carlo simulations f the GENIUS project for its application in double beta decay we refer to 2938 .

\subsubsection{GENIUS and Other Beyond Standard Model Physics}

HVKK-Triest01-Prepr: submitted to World Scientific on November 10, 201811 
GENIUS will allow besides the large increase in sensitivity for double beta decay described above, the access to a broad range of other beyond SM physics topics in the multi- $\mathrm{TeV}$ range. Already now $\beta \beta$ decay prohes the $\mathrm{TeV}$ scale on which new physics should manifest itself (see, e.g.12.28,40.41.37). Basing to a large extent on the theoretical work of the Heidelberg group in the last six years, the HEIDELBERG-MOSCOW experiment yields results for SUSY models (R-parity breaking, neutrino mass), leptoquarks (leptoquarks-Higgs coupling), compositeness, right-handed $W$ mass, nonconservation of Lorentz invariance and equivalence principle, mass of a heavy left or righthanded neutrino, competitive to corresponding results from high-energy accelerators like TEVATRON and HERA (for details see $12,40,41$ ).

\subsubsection{GENIUS-Test Facility}

Construction of a test facility for GENIUS - GENIUS-TF - consisting of $\sim 40 \mathrm{~kg}$ of HP Ge detectors suspended in a liquid nitrogen box has been started. Up to summer of 2001, six detectors each of $\sim 2.5 \mathrm{~kg}$ and with a threshold as low as $\sim 500 \mathrm{eV}$ have been produced.

Besides test of various parameters of the GENIUS project, the test facility would allow, with the projected background of $2-4$ events/(kg y keV) in the low-energy range, to probe the DAMA evidence for dark matter by the seasonal modulation signature, (se 42,43 , and section 3 ).

\section{Dark Matter}

\subsection{SUSY Expectations for Cold Dark Matter}

Direct search for WIMPs can be done

(a) by looking for the recoil nuclei in WIMP- nucleus elastic scattering. The signal could be ionisation, phonons or light produced by the recoiling nucleus. The typical recoil energy is a few $100 \mathrm{eV} / \mathrm{GeV}$ WIMP mass.

(b) by looking for the modulation of the WIMP signal resulting from the seasonal variation of the earth's velocity against the WIMP 'wind'.

The expectation for neutralino elastic scattering cross sections and masses have been extensively analysed in many variants of SUSY models.

Figs.6, 7 represent the present situation. The SUSY predictions in Figs.6a, 7 are from the MSSM with relaxed unification conditions 66 and the MSUGRA model 6 . Fig. $6 \mathrm{~b}$ shows the result of a study 'at Post-LEP Benchmark points' based again on the MSUGRA68. Present experiments only just touch the border of the area predicted by the MSSM. The experimental DAMA evidence for dark matter lies in an area, in which MSUGRA mod-

HVKK-Triest01-Prepr: submitted to World Scientific on November 10, $2018 \mathbf{2}$ 


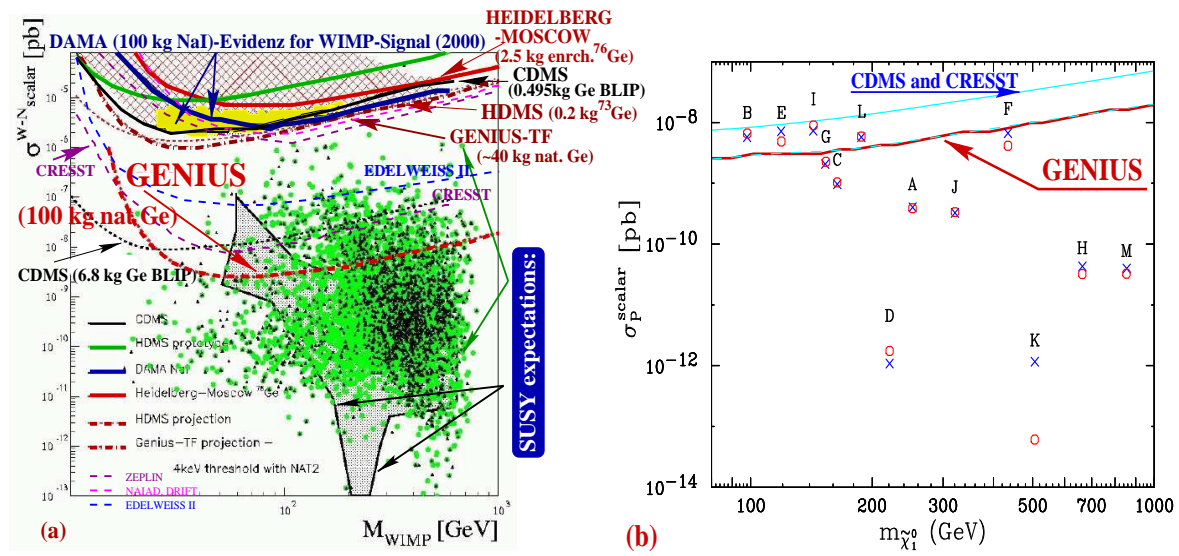

Figure 6. (a): WIMP-nucleon cross section limits in pb for scalar interactions as function of the WIMP mass in GeV. Shown are contour lines of present experimental limits (solid lines) and of projected experiments (dashed lines). Also shown is the region of evidence published by DAMA. The theoretical expectations from the MSSM-are shown by two scatter plots, for accelerating a for non-accelerating Universe (from 66 ), and from the SUGRA by the grey region (from67). Only GENIUS will be able to probe the shown range also by the signature from seasonal modulations. (b): WIMP- proter elastic scattering cross sections according to various MSUGRA models (see text). From68.

els do notexpect dark matter. They would require beyond GUT physics in this frame 69 .

It is clear from extensive theoretical work that high-sensitivity dark matter experiments can yield an important contribution to SUSY search. Fig.8 (from 70 ) shows, in the MSUGRA model, the SUSY reach contours for different accelerators (LEP2, Tevatron, LHC, NLC) together with direct detection rates in a ${ }^{73} \mathrm{Ge}$ detector. It is visible that a detector of GENIUS-sensitivity operates in SUSY search on the level of LHC and NLC. Fig.9 (from 66 ), shows another study in the MSSM with relaxed unification conditions. Non-observation of Dark Matter with GENIUS would exclude a 'light' SUSY spectrum (all sfermion masses lighter than $300-400 \mathrm{GeV}$ ) and any possibility for a light Higgs sector in the MSSM.

If classifying the SUGRA models into more $\mathrm{g}_{\mu}$-2-friendly (I,L,B,G,C,J) and less $\mathrm{g}_{\mu}$-2-friendly models, according to 68 , the former ones have good prospects to be detectable by LHC and/or a $1 \mathrm{TeV}$ collider. GENIUS could check not only the larger part of these ones, but in addition two of the less $\mathrm{g}_{\mu}$-2-friendly models ( $\mathrm{E}$ and $\mathrm{F}$ ), which will be difficult to be probed by fu-

HVKK-Triest01-Prepr: submitted to World Scientific on November 10, 201813 


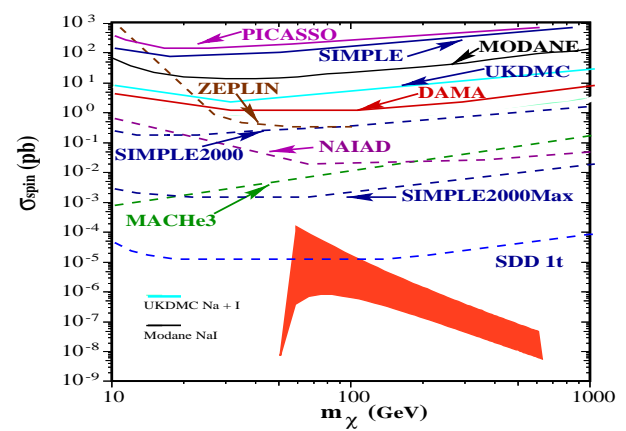

Figure 7. The same in Figs.6a, but forspin-dependent interaction. The grey area corresponds to the MSUGRA expectations67. Full lines denote present experimental results, dashed lines expectations of future experiments.

ture colliders (see Fig.6b). This demonstrates nicely the complementarity of collider and underground research.

It might be mentioned that in case of $\mathrm{g}_{\mu}-2$ - unfriendly models, i.e. those with very low cross sections in Figs.6b, it might be required to turn from spin-zero targets and looking for spin-independent interaction, which usually for not too light nuclei gives the largestcross sections, to spin- non-zero target nuclei and spin-dependent interaction 6 . It has been shown recently 66 that if spin-zero experiments with sensitivities of $10^{-5}-10^{-6}$ events $/ \mathrm{kg}$ day will fail to detect a dark matter signal, an experiment with nonzero spin target and higher sensitivity will be able to detect dark matter only due to the spin neutralino-quark interaction (see Fig.10).

\subsection{Cold Dark Matter - Present and Future Experiments}

Summarizing the present experimental status, present and also future projects can be categorized in two classes:

1. Sensitivity (or sensitivity goal) 'just for' confirmation of DAMA.

2. Sensitivity to enter deeply into the range of SUSY predictions.

Only very few experiments may become candidates for category 2 in a foreseeable future (see Figs.6.67), and as far as at present visible, of those only

HVKK-Triest01-Prepr: submitted to World Scientific on November 10, 201814 

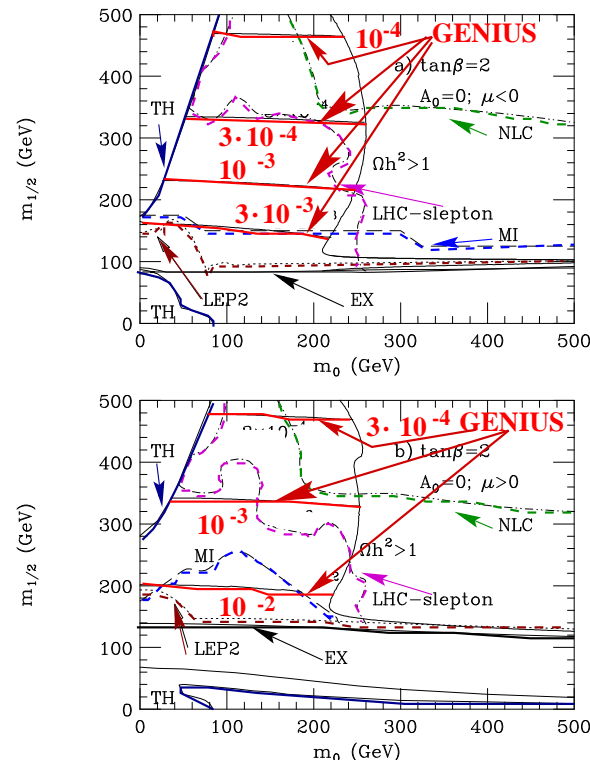

Figure 8. Direct detection rate $\mathrm{R}$ in events/kg day in a ${ }^{73} \mathrm{Ge}$ detector, and SUSY reach contours for LEP2, Tevatron (MI), LHC slepton signal and NLC. TH - excluded hy theoretical considerations. EX - excluded by collider searches for SUSY particles (from 70 ).

GENIUS will have the chance to search for modulation, i.e. to check, like DAMA, positive evidence for a dark matter signal.

Figs.11,12 give an overview of present and future experiments. The at present most sensitive experiments DAMA, CDMS (and Edelweiss) are claimed 71 not to be fully consistent although CDMS can at present not exclude the full DAMA evidence region 73,11 . Some problems in the data analysis of CDMS have been revised recently 2 .

One of the main problem of the cryodetectors is to obtain good numbers of background in the raw data, i.e. of the starting values for the rejection procedure. This jsalso the reason, why simple cryogenic calorimeters such as the CRESST 182.84 and the Milano53 89 bolometers are not fully competitive.

The at present most sensitive experiments using raw data are the HEIDELBERG-MOSCOW experiment $\$$ and the HEIDELBERG Dark Matter Search Experiment (HDMS) 33 81. HDMS uses a special configuration of Ge detectors, with an enriched ${ }^{73} \mathrm{Ge}$ detector embedded into a patural Gedetector (Fig.13a). Results of the pilote project are given in 83 . 81 . The final configuration with the enriched ${ }^{73} G e$ inner detector is in operation since

HVKK-Triest01-Prepr: submitted to World Scientific on November 10, 201815 


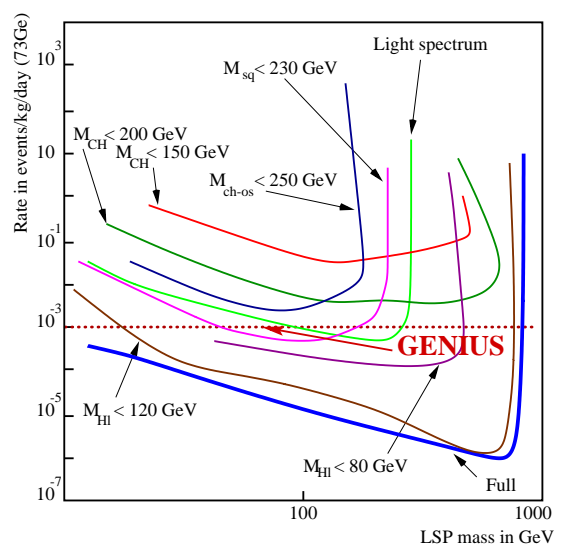

Figure 9. Different lower bounds for the total event rate in ${ }^{73} \mathrm{Ge}$ (events/day kg) versus mass of the LSP $(\mathrm{GeV})$. Here $\mathrm{M}_{s q, C H, H l}$ denote masses of the squark, the charged Higgs boson, and the light neutral CP-even HIggs boson, respectively. Heavy chargino mass is denoted as $\mathrm{M}_{c h-o s}$. "Full" corresponds to the lower bound obtained from main (unconstrained) scan, and "light spectrum" denotes the lower bound for R, which is obtained with all sfermion masses lighter then about $300 \mathrm{GeV}$. The horizontal dotted lime represents expected sensitivity for the direct dark matter detection with GENIUS (from66).

February 2001.

The experimental future is also illustrated in Figs.6, 6. A useful overview is given in 64, and for Ge detectors in 79 . For an earlier review see 87 .

The cryogenic projects are CDMS II, CREST II, Edelweiss II. In contrast to CDMS and Edelweiss which do its active background rejection by looking for ionisation and phonons, CRESST II plans to use simultaneous detection of light and phonons. CDMS II plans to use 42 detectors with a total mass of $6.8 \mathrm{~kg}$ of Ge by 2006 in the Soudan mine, CRESST II plans to have $10 \mathrm{~kg}$ of $\mathrm{CdWO}_{4}$ in the Gran Sasso in some future. The cryogenic experiments are, however, operating at present only $600 \mathrm{~g}$ of detectors or less, after a decade of development. CDMS has collected only $10.6 \mathrm{~kg} \mathrm{~d}$ of data over this time (in 1999, since then no measurement) 1 , Edelweiss only $4.53 \mathrm{~kg}$ d 75 . Therefore, they may have severe problems to expand their small detector masses to several tens of $\mathrm{kg}$ or better $100 \mathrm{~kg}$, as required for modulation search. This means that although e.g. CDMS II may reach a future sensitivity in an exclusion plot as shown in Fig. $6 \mathrm{~b}$ b, it will not be able to look for the modulation signal. A general problem in the present stage still seems to be the reproducibility of the highly complicated cryo detectors. In spite of this, phantasy is large enough, to dream already about 1 ton cryo detectors systems 11 .

HVKK-Triest01-Prepr: submitted to World Scientific on November 10, 201816 

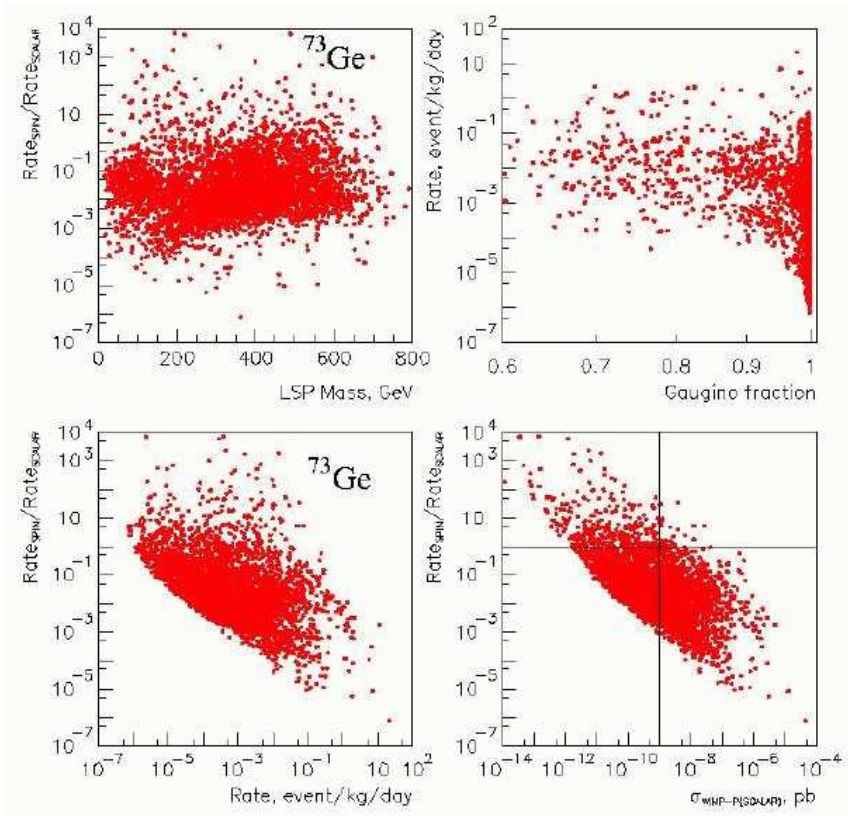

Figure 10. Ratio of spin-dependent event rate to spin-independent event rate in ${ }^{73} \mathrm{Ge}$ as function of LSP mass (upper left), of total (spin-dependent plus spin-independent) event rate (lower left part), and of scalar cross section of neutralino-proton interaction (lower right) obtained with $0.1<\Omega_{\chi} h_{0}^{2}<0.3$. The vertical line gives the expected sensitivity of GENIUS. In the region above the horizontal line the spin contribution domintes. The total event rate versus gaugino fraction of LSP is also given (upper right). From 66.

Other far future projects are the superheavy droplet detectors PICASSO/SIMPLE64. They are working at present on a scale of 15 and $50 \mathrm{~g}$ detectors. Their idea is to use $10-100 \mu \mathrm{m}$ diameter droplets of volatile $\mathrm{C}_{4} \mathrm{~F}_{10}, \mathrm{C}_{3} \mathrm{~F}_{8}, \ldots$ in metastable superheated condition and to choose critical energy and radius such that only nuclear recoils can trigger a phase transition, but not $\gamma$ and $\beta$ particles. The acoustic signal of the explosive bubble formation will be observed. The expected sensitivity of a 1 ton module for spin-dependent WIMP-nucleon interaction is shown in Figure $6 \mathrm{~b}$ - as SDD 1 ton (for an assumed U/Th contamination of $10^{-15} \mathrm{~g} / \mathrm{g}-\mathrm{U} / \mathrm{Th} \alpha$-emitters can cause recoil events!). A drawback is that these detectors cannot measure energy spectra of WIMPs.

A very promising project which would yield a nice signal identification, is DRIFT in the Boulby mine. It is aiming at looking for the diurnal directional

HVKK-Triest01-Prepr: submitted to World Scientific on November 10, 201817 


\begin{tabular}{|c|c|c|c|c|}
\hline NAME & TARGET & MASS (kg) & MODULATIONS & UPPER LIMIT \\
\hline DAMA-I & $\operatorname{NaI}(\mathrm{Tl})$ & 100. & YES $\begin{array}{r}2-20 \mathrm{keV} \\
57986.0 \mathrm{~kg} \mathrm{day}\end{array}$ & $\begin{array}{c}+ \\
\text { Runs since } 1996\end{array}$ \\
\hline DAMA-II & $\mathrm{NaI}(\mathrm{Tl})$ & 250. & YES & Plans for 2002 \\
\hline HDMS & ${ }^{73} \mathrm{Ge}$ & 0.2 & NO $\begin{array}{c}0.07 \mathrm{ev} / \mathrm{kg} \mathrm{d} \mathrm{keV} \\
40-100 \mathrm{keV} \\
0.2 \mathrm{ev} / \mathrm{kg} \mathrm{d} \mathrm{keV} \\
11-40 \mathrm{keV}\end{array}$ & Runs since 2001 \\
\hline GENIUS-TF & ${ }^{76} \mathrm{Ge}$ & 40. & $\begin{array}{l}\text { YES } \begin{array}{r}6 \text { new det. } \\
2.5 \mathrm{~kg} \\
\text { threshold } 500 \mathrm{eV} \\
\text { !!! }\end{array}\end{array}$ & $\begin{array}{l}\text { In construction } \\
\text { start in } 2002\end{array}$ \\
\hline GENIUS & $76 \mathrm{Ge}$ & $\begin{array}{r}100 . \\
1000 .\end{array}$ & $\begin{array}{l}\text { YES } \\
\text { YES }\end{array}$ & $\begin{array}{l}\text { Proposal since } 1997 \\
\text { Proposal since } 1997\end{array}$ \\
\hline CDMS-I & Ge (blip) & $\begin{array}{l}0.495(4 \times 0.165 \\
3 \text { intern, } 1 \text { anticoin. }\end{array}$ & $\begin{array}{l}\mathrm{NO} \quad 10-100 \mathrm{keV} \\
50 \text { (int) } \\
230 \text { (ext.) } \mathrm{kg}^{-1} \mathrm{~d}^{-1}\end{array}$ & $\begin{array}{l}\text { Run } 96 \text { live days in } \\
\text { period: } 11.98-09.99\end{array}$ \\
\hline CDMS-II & Ge (blip) & 10.6 & NO & Plan for 2006 \\
\hline CRESST-I & $\mathrm{CaWO}_{4}$ & 1.048 & $\begin{array}{l}\text { NO } 1.2 \mathrm{c} / \mathrm{keV} \mathrm{kg} \mathrm{d} \\
\text { at } 15 \mathrm{keV}\end{array}$ & Run in 1998 some \\
\hline CRESST-II & $\mathrm{CaWO}_{4}$ & 10. & NO & Plans since 2000 \\
\hline
\end{tabular}

Figure 11. Status of some running and future Dark Matter experiments.

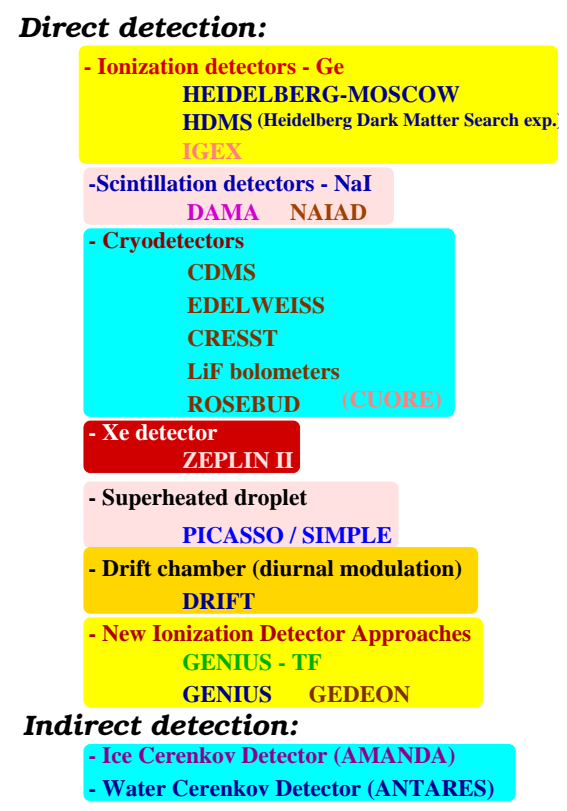

Figure 12. List of some of the main present and future dark matter experiments. 
modulation (Fig.13b). The idea is to detect tracks of nuclear recoils in a $\mathrm{TPC}$ with $\mathrm{Xe}(\mathrm{Ar})$ by a multiwire read-out. A $1 \mathrm{~m}^{3}$ prototype $(1.5 \mathrm{~kg} \mathrm{Xe})$ is under construction. It is seen as first component of the full $10 \mathrm{~m}^{3}$ DRIFT experiment 64.65 .

The ZEPLIN project uses scintillation and electro-luminescence in twophase xenon. Plans for ZEPLIN II are 0.01 - 0.1 counts $/ \mathrm{kg} \mathrm{d}$ and $20 \mathrm{~kg}$ of Xe. While still waiting for results of ZEPLIN I, plans are already discussed for a ZEPLIN IV76.

To return to the more 'earth-bound' projects: DAMA willextend their mass to $250 \mathrm{~kg}$, and plans to start operation in summer 2002 73 . Also the NAIAD project (Boulby mine)plans to use NaI - 40-100 kg of NaI in a liquid scintillator Compton veto 7 . The projected NAIAD limits for $100 \mathrm{~kg} y$ exposure are $0.1 \mathrm{c} / \mathrm{kgd}$. Because of the large mass it will be able to look for modulation.

The HDMS experiment and the GENIUS-TF experiment 42,43 aim at probing the DAMA evidence (see Fig.15). GENIUS-TF consisting of $40 \mathrm{~kg}$ of Ge detectors in liquid nitrogen (Fig.14a) could also measure the modulation signal 43 .78. Up to summer 2001, already 6 detectors of $2.5 \mathrm{~kg}$ each, with an extreme low-energy threshold of $\sim 500 \mathrm{eV}$ have been produced. A similar potential is aimed at by the GEDEON project 79 , which plans to use 28 Ge diodes in one single cryostat.

GENIUS-TF is already under installation in the Gran-Sasso laboratory and should start operation by end of 2002 .

The probably most far reaching project is GENIUS 2229. Since it is based on conventional techniques, using Ge detectors in liquid nitrogen, is may be realized in the most straightforward way.

GENIUS would already in a first step, with $100 \mathrm{~kg}$ of natural Ge detectors in three years of measurement, cover a significant part of the SUSY parameter space for prediction of neutralinos as cold dark matter (Figs. 6.77). For this purpose the background in the energy range $<100 \mathrm{keV}$ has to be reduced to $10^{-2}$ (events/ kg y keV). At this level solar neutrinos as source of background are still negligible. Of particular importance is to shield the detectors during production (and transport) to keep the packground from spallation by cosmic rays sufficiently low (for details see 39.85 .86).

The sensitivity of GENIUS for Dark Matter corresponds to that obtainable with a $1 \mathrm{~km}^{3}$ AMANDA detector for indirect detection (neutrinos from annihilation of neutralinos captured at the Sun) (see92). Interestingly both experiments would probe different neutralino compositions: GENIUS mainly gaugino-dominated neutralinos, AMANDA mainly neutralinos with comparable gaugino and Higgsino components (see Fig. 38 in 92 ).

HVKK-Triest01-Prepr: submitted to World Scientific on November 10, $2018 \mathbf{9}$ 

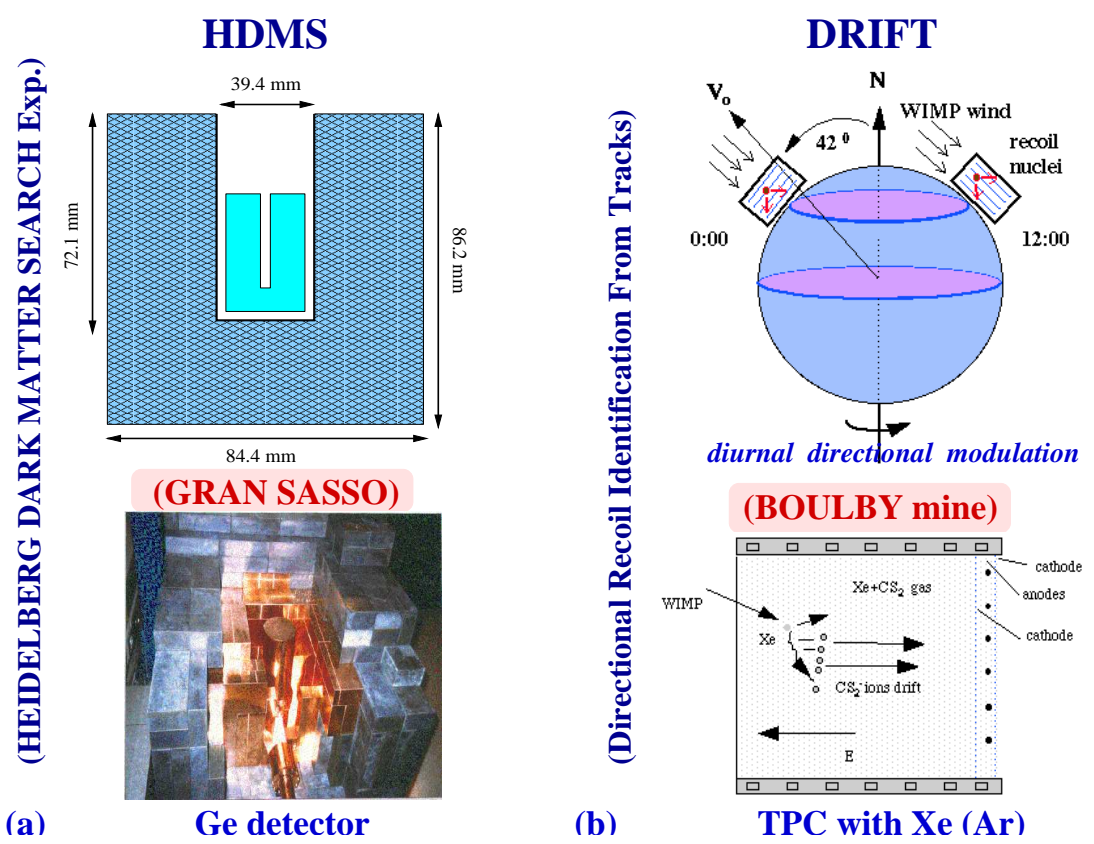

Figure 13. HDMS and DRIFT experiments.

\subsection{Hot Dark Matter Search}

According to the recent indication for the neutrinoless mode of double beta decayl, (see section 2), neutrinos should still play an important role as hot dark matter in the Universe.

The effective mass has been determined to bed $\langle m\rangle=(0.05-0.84) \mathrm{eV}$ at a $95 \%$ c.l. (best value $0.39 \mathrm{eV}$ ) including an uncertainty of $\pm 50 \%$ of the nuclear matrix elements.

With the limit deduced for the effective neutrino mass, the HEIDELBERG-MOSCOW experiment excludes several of the neutrino mass scenarios allowed from present neutrino oscillation experiments (see Fig.16) allowing mainly only for degenerate and partially degenerate mass scenarios and an inverse hierarchy $3 \nu$ - scenario (the latter being, however, strongly disfavored by a recent analysis of SN1987A). In particular hierarchical mass schemes are excluded.

Assuming the degenerate scenarios to be realized in nature we fix - according to the formulae derived in 10 - the common mass eigenvalue of the

HVKK-Triest01-Prepr: submitted to World Scientific on November 10, $2018 \mathbf{2 0}$ 

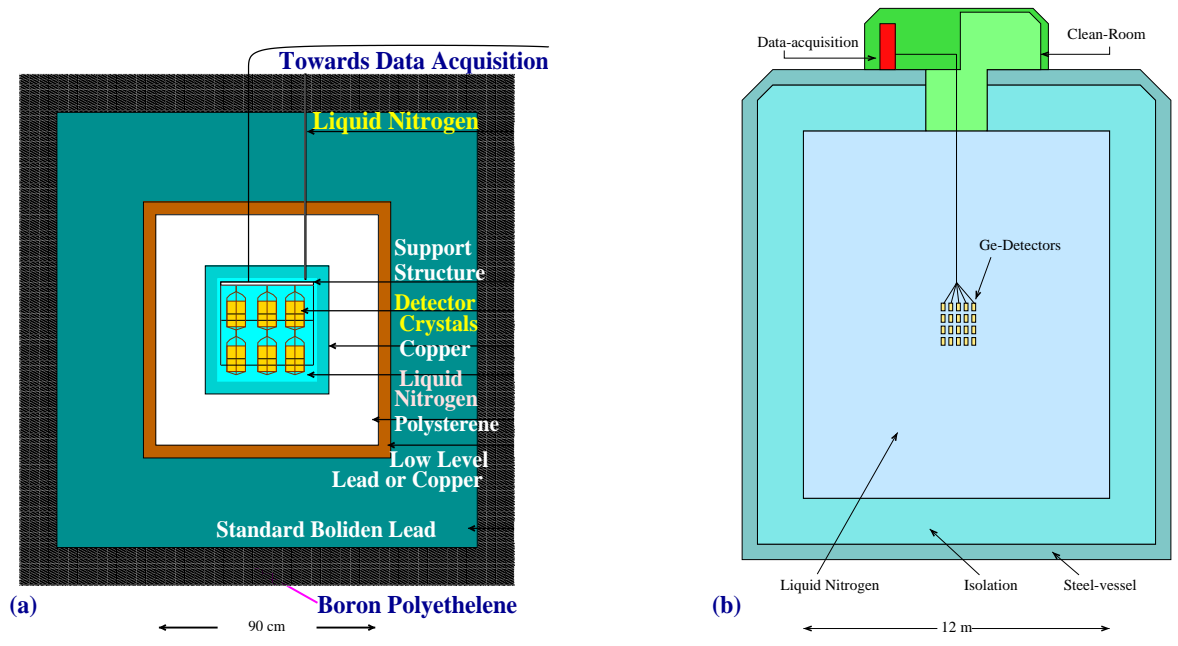

Figure 14. (a): Conceptual design of the Genius TF. Up to 14 detectors will be housed in the inner detector chamber, filled with liquid nitrogen. As a first shield $5 \mathrm{~cm}$ of zone refined Germanium, or extremely low-level copper w ill be used. Behind the $20 \mathrm{~cm}$ of polystyrene isolation another $35 \mathrm{~cm}$ of low level lead and a $15 \mathrm{~cm}$ borated polyethylene shield will complete the setup. nitrogen tank (

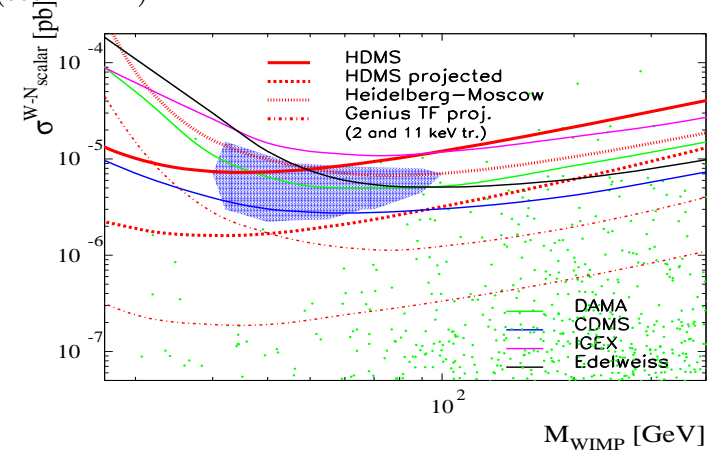

Figure 15. The potential of GENIUS-TF for dark matter search. WIMP-nucleon cross section limits as a function of the WIMP mass for spin-independent interactions. The solid lines are current limits of the HEIDELBERG-MOSCOW experiment, the HDMS, the DAMA and the CDMS experiments. The dashed curves are the expectation for HDMS, and for Genius-TF with an energy threshold of $11 \mathrm{keV}$ and $2 \mathrm{keV}$ (no tritium contamination) respectively, and a background index of 2 events $/ \mathrm{kg}$ y keV below $50 \mathrm{keV}$. The filled contour represents the evidence region of the DAMA experiment

HVKK-Triest01-Prepr: submitted to World Scientific on November 10, 201821 


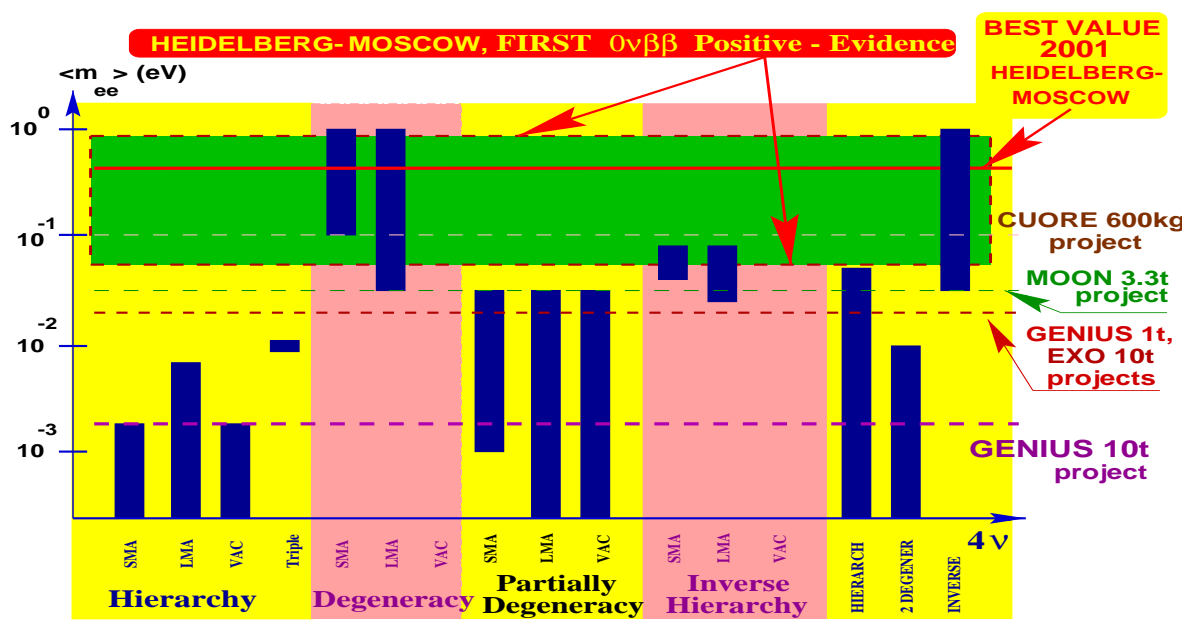

Figure 16. The impact of the evidence obtained for neutrinoless double beta decayl (best value of the effective neutrino mass $\langle m\rangle=0.39 \mathrm{eV}, 95 \%$ confidence range $(0.05-0.84) \mathrm{eV}$ allowing already for an uncertainty of the nuclear matrix element of a factor of $\pm 50 \%$, on possible neutrino mass schemes. The bars denote allowed ranges of $\langle m\rangle$ in different neutrino mass scenarios, still allowed by neutrino oscillation experiments. Hierarchical models are excluded by the new $0 \nu \beta \beta$ decay result. Also shown are the expected sensitivities for the future potential do perinents CUORE, MOON, EXO, and the 1 ton and 10 ton project of GENIUS29] 3688 (from 85 ).

degenerate neutrinos to $\mathrm{m}=(0.05-3.4) \mathrm{eV}$. Part of the upper range is already excluded by tritium experiments, which give a limit of $\mathrm{m}<2.2 \mathrm{eV}(95 \%$ c.l.) 2 . The full range can only partly (down to $\sim 0.5 \mathrm{eV}$ ) be checked by future tritium decay experiments, but could be checked by some future $\beta \beta$ experiments (see, e.g.12 80.65 ). The deduced best value for the mass is consistent with expectations from experimental $\mu \rightarrow e \gamma$ branching limits in models assuming the generating mechanism for the neutrino mass to be also responsible for the recent indication for as anomalous magnetic moment of the muon 61 . It lies in a range of interest also for Z-burst models recently discussed as explanation for super-high energy cosmic ray events beyond the GKZ-cutoffen. The sensitivity of the present result is already in the range to be probed by the satellite experiments MAP and PLANCK (Fig.17).

\section{Conclusion}

Dark matter search is presently one of the most exciting fields of particle physics and cosmology. Underground experiments at present only marginally

HVKK-Triest01-Prepr: submitted to World Scientific on November 10, $2018 \mathbf{2}$ 
$\mathrm{m}_{1}(\mathrm{eV})$ HEIDELBERG-MOSCOW Positive EVIDENCE

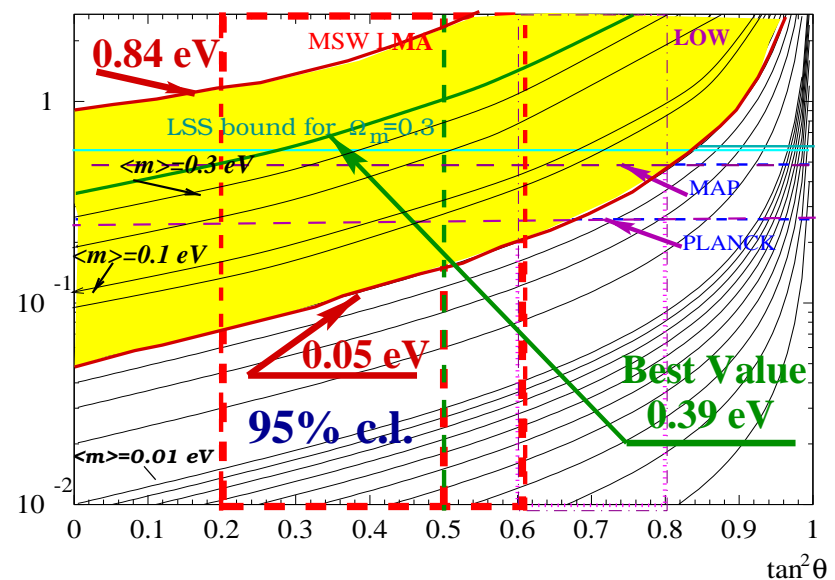

Figure 17. Double beta decay observable $\langle m\rangle$ and oscillation parameters: The case for degenerate neutrinos. Plotted on the axes are the overall scale of neutrino masses $m_{0}$ and mixing $\tan ^{2} \theta_{12}$. Atso shown is a cosmological bound deduced from a fit of CMB and large scale structure 31 and the expected sensitivity of the experiments MAP and PLANCK. The present limit from tritium $\beta$ decay of $2.2 \mathrm{eV} 64$ would lie near the top of the figure. The range of $\langle m\rangle$ fixed by the HEIDELBERG-MOSCOW experiment is, in the case of small solar neutrino mixing, already in the range to be explored by MAP and PLANCK.

touch in their sensitivity the range of present SUSY predictions for cold dark matter. Of future experiments the GENIUS project has the best prospects to cover a large part of the predicted range. GENIUS will provide information complementary to future collider search. This information is indispensable, even if LHC would find supersymmetry, since in any case it still has to be shown that SUSY particles indeed form the cold dark matter in the Universe. GENIUS will simultaneously be the most straightforward way to fix the neutrino mass and the contributions of neutrinos to hot dark matter with higher accuracy.

\section{References}

1. H.V. Klapdor-Kleingrothaus et al.,(HEIDELBERG-MOSCOW Collaboration), Eur. Phys. J A 12 (2001) 147 - 154 and hep-ph/0103062 and in Proc. of DARK2000, H.V. Klapdor-Kleingrothaus (Ed.), Springer-Verlag, Heidelberg, (2001) 520-533 and http://www.mpi-hd.mpg.de/non_acc/

2. E. Majorana, Nuovo Cimento 14 (1937) 171 - 184.

HVKK-Triest01-Prepr: submitted to World Scientific on November 10, $2018 \mathbf{2 3}$ 
3. G. Racah, Nuovo Cimento 14 (1937) 322 - 328.

4. W.H. Furry, Phys. Rev. 56 (1939) 1184 - 1193.

5. J. A. Mclennan, Jr. Phys. Rev. 106 (1957) 821.

6. K. M. Case, Phys. Rev. 107 (1957) 307.

7. D. V. Ahluwalia, Int. J. Mod. Phys. A 11 (1996) 1855.

8. H.V. Klapdor-Kleingrothaus, A. Dietz, H.L. Harney and I.V. Krivosheina; Modern Physics Letters A 16, No. 37 (2001) 2409-2420.

9. H.V. Klapdor-Kleingrothaus and U. Sarkar, Modern Physics Letters A 16, No. 38 (2001) 2469-2482.

10. H.V. Klapdor-Kleingrothaus, H. Päs and A.Yu. Smirnov, Phys. Rev. D 63 (2001) 073005 and hep-ph/0003219.

11. H.V. Klapdor-Kleingrothaus, H. Päs and A.Yu. Smirnov, in Proc. of DARK'2000, Heidelberg, 10-15 July, 2000, Germany, ed. H.V. Klapdor-Kleingrothaus, Springer, Heidelberg (2001) 420 - 434.

12. H.V. Klapdor-Kleingrothaus, "60 Years of Double Beta Decay - From Nuclear Physics to Beyond the Standard Model", World Scientific, Singapore (2001) $1281 \mathrm{p}$.

13. R.N. Mohapatra and P.B. Pal, "Massive Neutrinos in Physics and Astrophysics", Singapore, Singapore: World Scientific, World Scientific lecture notes in physics, 41 (1991) $318 \mathrm{pp}$.

14. M. Doi et al., Prog. of Theor. Phys. Suppl. 83 (1985) 1-175.

15. K. Muto and H.V. Klapdor, in "Neutrinos", Graduate Texts in Contemporary Physics", ed. H.V. Klapdor, Berlin, Germany: Springer (1988) 183 - 238.

16. K. Grotz and H.V. Klapdor, "Die Schwache Wechselwirkung in Kern-, Teilchenund Astrophysik", B.G. Teubner, Stuttgart (1989), "The Weak Interaction in Nuclear, Particle and Astrophysics", IOP Bristol (1990), Moscow, MIR (1992) and China (1998).

17. H.V. Klapdor-Kleingrothaus and A. Staudt, "Teilchenphysik ohne Beschleuniger", B.G. Teubner, Stuttgart (1995), "Non-Accelerator Particle Physics", IOP Publishing, Bristol and Philadelphia (1995) and 2. ed. (1998) and Moscow, Nauka, Fizmalit (1998), translated by V.A. Bednyakov.

18. P.Vogel in "Current Aspects of Neutrino Physics", ed. D.O. Caldwell, Berlin, Heidelberg, Germany: Springer (2001) 177 - 198.

19. J. Hellmig and H.V. Klapdor-Kleingrothaus, Nucl. Instrum. Meth. $\mathbf{A} \mathbf{4 5 5}$ (2000) $638-644$.

20. J. Hellmig, F. Petry and H.V. Klapdor-Kleingrothaus, Patent DE19721323A.

21. B. Majorovits and H.V. Klapdor-Kleingrothaus. Eur.Phys.J. A6 (1999) 463.

22. G. D'Agostini, hep-ex/0002055, W. von der Linden and V. Dose, Phys. Rev. E 596527 (1999), and F.H. Fröhner, JEFF Report 18 NEA OECD (2000) and Nucl. Sci. a. Engineering 126 (1997) 1, K. Weise and W. Wöger, "Messunsicherheit und Messdatenauswertung", Wiley-VCH, Weinheim 1999, and P.M. Lee, "Bayesian Statistics: An Introduction", Second edition, Arnold, London 1997, and A. O'Hagan, "Bayesian Inference", Volume 2B of "Kendall's Ad-

HVKK-Triest01-Prepr: submitted to World Scientific on November 10, 201824 
vanced Theory of Statistics", Arnold, London, 1997.

23. A.Staudt, K.Muto and H.V.Klapdor-Kleingrothaus, Eur. Lett. 13 (1990) 31.

24. T. Tomoda, Rept. Prog. Phys. 54 (1991) 53 - 126.

25. R.B. Firestone and V.S. Shirley, Table of Isotopes, 8-th Edition, John Wiley and Sons, Incorp., N.Y. (1998).

26. G. Douysset et al., Phys. Rev. Lett. 86 (2001) 4259 - 4262.

27. J.G. Hykawy et al., Phys. Rev. Lett. 67 (1991) 1708.

28. H.V. Klapdor-Kleingrothaus in Proc. of BEYOND'97, Castle Ringberg, Germany, 8-14 June 1997, eds. by H.V. Klapdor-Kleingrothaus and H. Päs, IOP Bristol (1998) 485 - 531.

29. H.V. Klapdor-Kleingrothaus et al. MPI-Report MPI-H-V26-1999 and Preprint: hep-ph/9910205 and in Proc. of BEYOND'99, Castle Ringberg, Germany, 6-12 June 1999, edited by H.V. Klapdor-Kleingrothaus and I.V. Krivosheina, IOP Bristol (2000) 915 - 1014.

30. H.V. Klapdor-Kleingrothaus, in Proc. of NEUTRINO 98, Takayama, Japan, 4-9 Jun 1998, (eds) Y. Suzuki et al. Nucl. Phys. Proc. Suppl. 77 (1999) 357.

31. R.E. Lopez, astro-ph/9909414; J.R. Primack and M.A.K. Gross, astroph/0007165; J.R. Primack, astro-ph/0007187; J. Einasto, in Proc. of DARK2000, Heidelberg, Germany, July 10-15, 2000, Ed. H.V. KlapdorKleingrothaus, Springer, Heidelberg (2001) 3-11.

32. Y. Suzuki in Proc. of NEUTRINO2000, ed. J. Law et al. (2001), Nucl. Phys. B 91 (2001) $29-35$.

33. M.C. Gonzalez-Garcia et al., Phys. Rev. D 63 (2001) 033005.

34. HEIDELBERG-MOSCOW Coll. (M. Günther etal.), Phys.Rev.D55 (1997) 54.

35. HEIDELBERG-MOSCOW Coll., Phys. Rev. Lett. 83 (1999) 41 - 44.

36. H.V. Klapdor-Kleingrothaus, in Proc. of Int. Conference NOW2000 - "Origins of Neutrino Oscillations", ed. G. Fogli, Nucl. Phys. B 100 (2001) 309 - 313.

37. H.V. Klapdor-Kleingrothaus, Int. J. Mod. Phys. A 13 (1998) 3953.

38. H.V.Klapdor-Kleingrothaus, J.Hellmig, M.Hirsch, J. Phys. G 24 (1998) 483.

39. H.V. Klapdor-Kleingrothaus, in Proc. of Advances in Nuclear Physics, eds.: D. Poenaru and S. Stoica, World Scientific, Singapore (2000) 123 - 129.

40. H.V. Klapdor-Kleingrothaus, in Proc. of 'Lepton-Baryon98', Trento, Italy, 2025 April, 1998, ed. H.V. Klapdor-Kleingrothaus and I.V. Krivosheina, IOP, Bristol, (1999) 251-301 and Preprint: hep-ex/9901021.

41. H.V. Klapdor-Kleingrothaus, Springer Tracts in Modern Physics, 163 (2000) 69-104, Springer-Verlag, Heidelberg, Germany (2000).

42. H.V. Klapdor-Kleingrothaus, L.Baudis, A.Dietz, G.Heusser, I.Krivosheina, B.Majorovits and H. Strecker, Internal Report MPI-H-V32-2000.

43. H.V. Klapdor-Kleingrothaus et al., hep-ph/0103082, subm. for publ. (2001).

44. A. Faessler and F. Simkovic, J. Phys. G 24 (1998) 2139 - 2178.

45. W.C. Haxton and G.J. Stephenson, Prog. Part. Nucl. Phys. 12 (1984) 409.

46. X.R. Wu, A. Staudt, H.V. Klapdor-Kleingrothaus, Cheng-Rui Ching and TsoHsiu Ho, Phys. Lett. B 272 (1991) 169 - 172.

HVKK-Triest01-Prepr: submitted to World Scientific on November 10, $2018 \mathbf{2 5}$ 
47. X.R. Wu, A. Staudt, T.T.S. Kuo and H.V. Klapdor-Kleingrothaus, Phys. Lett. B 276 (1992) $274-278$.

48. H. Ejiri et al., Phys. Rev. Lett. 85 (2000) 2917-2920.

49. M. Danilov et al., Phys. Lett. B 480 (2000) 12 - 18.

50. G. Gratta in Proc. of LowNu2, Dec. 4-5 (2000) Tokyo, Japan, ed: Y. Suzuki, World Scientific (2001).

51. T. Bernatowicz et al., Phys. Rev. Lett. 69 (1992) $2341-2344$

52. F.A. Danevich et al., Phys. Rev. C 62 (2000) 045501.

53. A. Alessandrello et al., Phys. Lett. B 486 (2000) 13-21 and S. Pirro et al., Nicl. Instr. Methods A 444 (2000) 71-76.

54. V.I. Tretyak and Yu.G. Zdesenko, At. Data Nucl. Data Tables 61 (1995) 43.

55. NEMO Coll., Contr. paper for XIX Int. Conf. NEUTRINO2000, Sudbury, Canada, June 16 - 21, 2000 LAL 00-31 (2000) 1 - 10 and in Proc. of NANPino2000, Dubna, Russia, July 2000, ed. V. Bednjakov et al. (2001) 1296.

56. E. Fiorini in Proc. of NEUTRINO2000, ed. J. Law et al. Nucl. Phys. B 91 (Proc. Suppl.) (2001) 262 - 269.

57. C.E. Aalseth et al. (IGEX Collaboration), Yad. Fiz. 63, No 7 (2000) 1299.

58. L. DeBraekeleer, talk at Work.. on the Next Gener. U.S. Undergr. Sci. Fac., WIPP, June 12-14, 2000, Carlsbad, New Mexico, USA and C.E. Aalseth et al., in Proc. of TAUP'2001, Gran Sasso, Italy September 2001 ed. A. Bettini.

59. Proc. Int. Workshop on Low Energy Solar Neutrinos, LowNu2, December 4 and 5 (2000) Tokyo, Japan, ed: Y. Suzuki, World Scientific, Singapore (2001).

60. G. Bellini et al. Phys. Lett. B 493216 - 228 (2000).

61. E. Ma and M. Raidal, Phys. Rev. Lett. 87 (2001) 011802; Erratum-ibid. 87 (2001) 159901.

62. K. Freese et al. in Proc. of DARK'98, Heidelberg, July 1998, IOP, Bristol (1999) eds. H.V. Klapdor-Kleingrothaus et al. 352; D. Graff, in Proc. of DARK 2000, Heidelberg, July 2000, Springer, Heidelberg (2001) ed. H.V. Klapdor-Kleingrothaus, 352 and K. Freese et al. in Proc. of DM'2000, WS (2001) 213 - 223 and astro-ph/0007444.

63. S. Asztalos et al., Phys. Rev. D 64 (2001) 092003; G.G. Raffelt in Proc. of DARK'2000, Heidelberg, July 2000, Springer, Heidelberg (2001), ed. H.V. Klapdor-Kleingrothaus, 60.

64. V. Zacek NPB, in Proc. of NEUTRINO2000, Sudbury, Canada, June 2000, ed. J. Law et al. (2001), Nucl. Phys. Proc. Suppl. 91 (2001) 368.

65. M.J. Lehner et al., in Proc.of DARK'2000, Heidelberg, July 2000, Springer, Heidelberg (2001), ed. H.V. Klapdor-Kleingrothaus, 590-597, C.J. Martoff, DRIFT Collab. in Proc. York 2000, ed. J.C. Spooner et al. 463-474.

66. V.A. Bednyakov and H.V. Klapdor-Kleingrothaus, Phys. Rev. D 62 (2000) 043524/1, and Phys. Rev. D 63 (2001) 095005.

67. J. Ellis et al. Phys.Lett. B481 (2000) 304 and Phys.Rev. D63 (2000) 065016.

68. J. Ellis et al., hep-ph/0111294.

69. R. Arnowitt, private communication, 2001

HVKK-Triest01-Prepr: submitted to World Scientific on November 10, $2018 \mathbf{2 6}$ 
70. M. Brhlik in Proc. of DARK'98, Heidelberg, July 1998, IOP, Bristol (1999) eds. H.V. Klapdor-Kleingrothaus et al. 499-515.

71. R.J. Gaitskell, NANP2001, Dubna, Russia, June 2001, to be publ. in Nuclei, Letters (2002).

72. B. Sadoulet in Proc.TAUP'2001, Sept.2001, Gran Sasso, Italy, ed. A. Bettini.

73. R. Bernabei et al., in Proc.: of Erice'2001, Sept.2001, Erice, Italy, ed. A. Faessler and of TAUP'2001, Sept.2001, Gran Sasso, Italy, ed. A. Bettini.

74. F. Pröbst in Proc. of TAUP'2001, Sept. 2001, Gran Sasso, Italy, ed. A. Bettini.

75. J. Gascon et al. in Proc.of DARK'2000, Heidelberg, July 2000, Springer, Heidelberg (2001), ed. H.V. Klapdor-Kleingrothaus, 575-580 and A. de Lesquen et al. in Proc. of NANP2001, Dubna, Russia, June 2001, ed. V. Bednjakov, to be publ. in Nuclei, Letters (2002).

76. D.B. Cline, astro-ph/0111098.

77. J.C. Spooner in Proc. of York'2000, ed. J.C. Spooner et al. WS (2001).

78. H.V. Klapdor-Kleingrothaus et al., subm for publ. 2001.

79. A. Morales., hep-ex/0111089.

80. HEIDELBERG-MOSCOW Collaboration, Phys. Rev. D 59 (1998) 022001.

81. L. Baudis, A. Dietz, B. Majorovits, F. Schwamm, H. Strecker and H.V. Klapdor-Kleingrothaus, Phys. Rev. D 63 (2000) 022001.

82. Y. Ramachers for the CRESST Coll. in Proc. of XIth Renc. de Blois, Front. of Matter, France, June 27-July 3, 1999.

83. H.V. Klapdor-Kleingrothaus et al. in Proc. of DARK2000, Heidelberg, Germany, July 10-15, 2000, Springer, Heidelberg (2001), ed. H. V. Klapdor-Kleingrothaus 553- 568 .

84. T.J. Weiler, in Proc. Beyond the Desert 1999, Tegernsee, Germany, 6-12 Juni 1999, edited by H.V. Klapdor-Kleingrothaus and I.V. Krivosheina, IOP Bristol (2000) 1085 - 1106; H. Päs and T.J. Weiler, Phys. Rev. D 63 (2001) 113015.

85. H.V. Klapdor-Kleingrothaus, in Proc. of NOON2000, Tokyo, Dec. 2000, World Scientific, Singapore (2001).

86. M. Tegmark, M. Zaldarriaga and A.J.S. Hamilton, Preprint: hep-ph/0008145.

87. H.V. Klapdor-Kleingrothaus and Y. Ramachers, Eur.Phys.J. A3 (1998) 85-92.

88. H.V. Klapdor-Kleingrothaus, in Proc. of LowNu2, Dec. 4-5., Tokyo, Japan, ed: Y. Suzuki et al. World Scientific, Singapore (2001).

89. E. Fiorini et al., Phys. Rep. 307, 309 (1998).

90. A. Alessandrello et al., Phys. Lett. B 335, 519-525 (1994).

91. A. Giuliani (CUORE Collaboration), in Proc. Astrophysics and Cosmology', 'Lepton-Baryon98',eds. H.V. Klapdor-Kleingrothaus and I.V.Krivosheina, Trento, Italy, April 20 - April 25, 1998, IOP, Bristol, 302 - 308 (1999).

92. J. Edsjö, home page: http://www.physto.se/edsjo

93. H.V. Klapdor-Kleingrothaus, in Proc. of NANPino-2000, Dubna, July 19-22, 2000, Part. and Nucl. Lett. iss. 1/2 (2001) 20-39 and hep-ph/0102319.

94. C. Weinheimer, in Proc. of DARK2000, Heidelberg, Germany, 10-16 July, 2000, ed. H.V. Klapdor-Kleingrothaus, Springer, Heidelberg (2001) 513 - 519.

HVKK-Triest01-Prepr: submitted to World Scientific on November 10, $2018 \mathbf{2 7}$ 